\title{
Extraction of Transport Dynamics in AIGaN/GaN HFETs Through Free Carrier Absorption
}

\author{
YUH-RENN WU, ${ }^{1,3}$ JOHN M. HINCKLEY, ${ }^{2}$ and JASPRIT SINGH ${ }^{2}$ \\ 1.-Department of Electrical Engineering, Graduate Institute of Photonics and Optoelectronics, \\ National Taiwan University, Taipei 106, Taiwan. 2.-Department of Electrical Engineering and \\ Computer Science, University of Michigan, Ann Arbor, MI 48109, USA. 3.—e-mail: yrwu@cc.ee.ntu. \\ edu.tw
}

The importance of AlGaN/GaN heterostructure field-effect transistor (HFETs) in high-power high-frequency applications is now well established. However, detailed information on high-field mobilities, velocity-field relations, carrier temperature, and momentum and energy relaxation times are not available. In this paper we carry out theoretical simulations based on Monte Carlo techniques to show that transport dynamics can be effectively extracted through free carrier absorption. Using short pulses of infrared radiation, it is possible to obtain the velocity-field curve by fitting the absorption spectrum without heating the device. We show this by solving the classical transport equation and then verify the results through Monte Carlo simulations. With the model presented it would be possible to extract carrier dynamics from experimentally measured results. Our work suggests that free carrier absorption experiments on AlGaN/GaN HFETs would provide important transport information, which would be very useful in device design and modeling.

Key words: III-V Nitrides, AlGaN, GaN, HFET, free carrier absorption, Drude theory, carrier transport, infrared

\section{INTRODUCTION}

The AlGaN/GaN heterojunction field-effect transistor (HFET) has established itself as a key player in high-power high-frequency applications. The maximum $f_{\mathrm{T}}$ of AlGaN/GaN HFETs has reached $181 \mathrm{GHz}^{1}$ and the $f_{\text {max }}$ has been shown to be over $230 \mathrm{GHz}{ }^{2}$ However, the electron velocity extracted from these devices shows an overall lower velocity than what is expected. In general a number of transport-related issues remain unclear in GaN-based devices. Traditionally, short pulse measurements are used to avoid the self-heating and hot-phonon effects. However, since pulse widths are usually longer than nanoseconds, it is hard to exclude these effects. ${ }^{3,4}$

(Received July 30, 2007; accepted October 9, 2007;

published online November 1, 2007)
There are several methods to determine carrier dynamics in the semiconductors. A direct de short pulse measurement can provide the momentum relaxation time of carriers. However, it cannot provide information on the carrier energy and energy relaxation time. The contact resistances also influence the accuracy of measurements. Optical pump probe measurement can provide information of carrier relaxation time. However, the screening effects of electron-hole pairs under the strong dc bias will influence these experiments. Jiang et al. ${ }^{5}$ have proposed the use of long-wavelength absorption spectroscopy through free carriers to provide information on carrier temperature as well as momentum and energy relaxation time. Several experimental studies ${ }^{6-8}$ have also demonstrated the feasibility of measuring free carrier absorption spectrum to study the carrier dynamics.

In this paper, we use the Monte Carlo method to study free carrier absorption effects in GaN HFETs 
at different intensities and frequencies. We present the potential use of terahertz absorption spectroscopy to provide information on carrier temperature, momentum and energy relaxation times. A Monte Carlo based numerical simulation is used to study the free carrier absorption of sub-bandgap radiation in $\mathrm{AlGaN} / \mathrm{GaN}$ HFETs. In our simulation, we will study the absorption spectrum under different conditions: (1) we will study the absorption of the twodimensional electron gas (2DEG) of $\mathrm{AlGaN} / \mathrm{GaN}$ HFETs with different infrared intensities and frequencies; (2) we also examine ac absorption in the presence of a dc bias on the device and calculate the absorption spectrum of an infrared radiation at different frequencies and intensities. Jiang et al. ${ }^{5}$ proposed the idea of measuring the absorption spectrum of Si under dc bias to extract the energy relaxation time; and (3) we will compare the differences between the carrier absorption behaviors in GaN and GaAs HFETs.

Our approach is based on the following approach. We first develop a general model for carrier dynamics using a generalized Drude model. In this analytic model the carrier dynamics are expressed in terms of the momentum and energy relaxation times and carrier masses. We then fit the absorption calculated from the Monte Carlo calculations and extract the carrier dynamics parameters, which we compare with the values obtained directly from the Monte Carlo simulation. The closeness of these two approaches allows us to validate our analytical model.

\section{FORMALISM}

In this paper, we use the ensemble Monte Carlo (EMC) method that we have developed for device simulations ${ }^{9}$ to study and compare the free carrier absorption behavior in AlGaN/GaN HFETs and AlGaAs/GaAs HFETs. The scattering mechanisms accounted for in the simulation are (1) two- (2D) and three-dimensional (3D) polar optical phonon absorption and emission, (2) acoustic phonon scattering, (3) equivalent and nonequivalent intervalley scattering, (4) alloy scattering, (5) interface roughness scattering, (6) dislocation scattering, and (7) ionized impurity scattering. The material parameters are taken from Ref. 10. In the EMC method, an electron will absorb energy from the applying electric field during the free flight time $\tau$. Due to the scattering mechanisms mentioned above, the electron will have a different momentum and energy after scattering. The energy absorbed by the electron during the free flight period is mainly released by optical phonon emission. The energy absorbed can be expressed as

$$
E_{\mathrm{abs}}=E(k(t+\tau))-E(k(t)),
$$

$$
\hbar k(t+\tau)=\hbar k(t)+\frac{q F_{\mathrm{ac}}}{\omega}(\cos \omega t-\cos \omega(t+\tau)),
$$

where $q$ is the electronic charge, $E_{\text {abs }}$ is the energy absorbed by the free carrier, $\omega$ is the radiation frequency, and $F_{\text {ac }}$ is the field amplitude, where the infrared radiation source is expressed in the classical picture by

$$
F=F_{\text {ac }} \sin \omega t
$$

After a sufficient long simulation, energy balance is reached, i.e., the electron energy absorbed by the electric field is equivalent to the energy loss by scattering especially the optical phonon emission process. The absorption loss, $L$, by the 2 DEG can be expressed by

$$
L=\frac{E_{\mathrm{abs}} n_{2 \mathrm{DEG}}}{T N_{\mathrm{e}}} \frac{1}{I},
$$

where $N_{\mathrm{e}}$ is the number of particles being simulated, $n_{2 \text { DEG }}$ is the carrier density of the 2DEG $\left(\mathrm{cm}^{-2}\right)$, and $T$ is the simulation time. $E_{\text {abs }}$ is the total energy absorbed by electron and can be obtained from Eq. 1. $I$ is the input power density, which can be expressed by

$$
I=\frac{1}{2} c n \epsilon_{0} F_{\mathrm{ac}}^{2}
$$

where $c$ is the velocity of light, $n$ is the refractive index, and $\epsilon_{0}$ is the permittivity of vacuum. Note that here we are interested in studying the total energy absorbed by the 2DEG. Therefore, the units of $n_{2 \mathrm{DEG}}$ are $\mathrm{cm}^{-2}$. And the units of $L$ are percentage instead of $\mathrm{cm}^{-1}$. The strength of the electric field we used is from $1 \mathrm{kV} / \mathrm{cm}$ to $150 \mathrm{kV} / \mathrm{cm}$ because we are interested in the high-field mobility of GaN HFETs. The radiation frequency we studied is from $50 \mathrm{GHz}$ to $100 \mathrm{THz}$, which covers a wide range from millimeter to infrared sources. In the Monte Carlo program, two cases are studied. For the first case, only the strength and frequency of the radiation source are varied. For the second case, a dc bias is applied on the device. A linear polarized radiation source with different frequency is applied to the device as a small perturbation. Therefore, the strength of the radiation source should be much smaller than the dc bias. The polarized wave perpendicular and parallel to the dc bias are both studied.

It would be useful to compare the Monte Carlo results with a simple theoretical prediction to give us some insight into the physics. The free carrier absorption can be expressed by Drude-Zener theory. In the Drude-Zener theory, the relation between the absorption and electron relaxation time can be expressed by

$$
L=\frac{n_{2 \mathrm{DEG}} q^{2}}{m^{*} c n \epsilon_{0}} \frac{\left\langle\tau_{\mathrm{m}}\right\rangle}{1+\left(\omega\left\langle\tau_{\mathrm{m}}\right\rangle\right)^{2}},
$$

and 
where $\left\langle\tau_{\mathrm{m}}\right\rangle$ is the electron momentum relaxation time and $\omega$ is the radiation frequency. When $\omega\left\langle\tau_{\mathrm{m}}\right\rangle \gg 1$,

$$
L=\frac{n_{2 \mathrm{DEG}} q^{3}}{m^{* 2} \operatorname{cn} \epsilon_{0}} \frac{1}{\omega^{2} \mu},
$$

where $\mu$ is equal to $q\left\langle\tau_{\mathrm{m}}\right\rangle / \mathrm{m}^{*}$. The absorption loss will be the inverse of the electron mobility. On the other hand, when $\omega\left\langle\tau_{\mathrm{m}}\right\rangle \ll 1$,

$$
L=\mu \frac{n_{\mathrm{e}} q}{c n \epsilon_{0}},
$$

where the absorption loss is proportional to the electron mobility. When the device is under dc bias, as suggested in Ref. 5, the motion of the electrons can be expressed by

$$
\begin{gathered}
\frac{\mathrm{d} E}{\mathrm{~d} t}=q \mathbf{v} \cdot \mathbf{F}-\frac{E-3 / 2 k_{\mathrm{B}} T_{\mathrm{L}}}{\tau_{\mathrm{E}}} \\
\frac{\mathrm{d} \mathbf{p}}{\mathrm{d} t}=q \mathbf{F}-\frac{\mathbf{p}}{\tau_{\mathrm{m}}},
\end{gathered}
$$

where $E, \mathbf{p}, \tau_{\mathrm{E}}$, and $\tau_{m}$ are the carrier energy, momentum, energy relaxation time, and momentum relaxation time, respectively. $\mathbf{F}$ is the applied electric field, and $T_{\mathrm{L}}$ is the lattice temperature. When electrons are under steady-state conditions, we obtain

$$
\begin{gathered}
v=\frac{q \mathbf{F} \tau_{\mathrm{m}}}{m^{*}} \\
E=\frac{q^{2} \tau_{\mathrm{m}} \tau_{\mathrm{E}} F^{2}}{m^{*}}+\frac{3}{2} k_{\mathrm{B}} T_{\mathrm{L}} .
\end{gathered}
$$

$\tau_{m}$ and $\tau_{\mathrm{E}}$ are usually functions of energy and can be assumed to be $\tau_{\mathrm{m}} \propto 1 / E^{a}$ and $\tau_{E} \propto E^{b}$. Now if an infrared radiation source is incident onto the device, we can treat it as a small perturbation. We expand the equation at $\mathbf{F}=\mathbf{F}_{\mathrm{dc}}$ where $\mathbf{F}_{\mathrm{dc}}$ is the dc bias field and use the relation of $\tau_{m}$ and $\tau_{E}$ to $E$, we get where $v_{0}, \tau_{E 0}, \tau_{m 0}$ and $E_{0}$ are the values without perturbation, and $\Delta v$ and $\Delta E$ are the corresponding changes due to the light. $\theta$ is the angle between the dc bias field and the polarization of the radiation source. We can now obtained the power absorbed by a single carrier from the optical field:

$$
P=\frac{1}{2} \operatorname{Re}\left\{q \mathbf{F}_{\mathbf{a c}} \cdot \Delta v\right\} .
$$

When the polarization of the incident infrared source is perpendicular to the dc bias, $\cos \theta=0$. Therefore the absorption loss is

$$
L_{\perp}\left(F_{\mathrm{dc}}\right)=\mu_{\perp}\left(\boldsymbol{F}_{\mathrm{dc}}\right) \frac{n_{2 \mathrm{DEG} q}}{c n \epsilon_{0}} \frac{1}{1+\left(\omega \tau_{m}\right)^{2}} .
$$

When $F_{\mathrm{dc}}$ is equal to 0 , the above equation will be equivalent to the Drude-Zener theory.

With the polarization parallel to the dc bias and in the velocity saturation region, as suggested in Ref. 5, the absorption loss can be expressed by

$$
L_{\|}\left(F_{\mathrm{dc}}\right)=L_{\perp}\left(F_{\mathrm{dc}}\right) \frac{\left(\omega \tau_{E}\right)^{2}}{4 a^{2}+\left(\omega \tau_{E}\right)^{2}} .
$$

It is easy to see that $L_{\|}$reaches a maximum at $\omega=\sqrt{\tau_{E} \tau_{m}}$ in the velocity saturation region. If the electron is not biased at the velocity saturation region, Eq. 17 would not represent a good approximation since a number of simplifying assumptions have been made in deriving it ${ }^{5}$ and these are good approximations only in the saturation region. Therefore, we need to fit Eqs. 13 and 15 instead of Eq. 17 to extract $\tau_{E}$ and $\tau_{m}$. Note that this analytical model might be oversimplified to explain the results from Monte Carlo or experimental results. To explain the experimental results exactly, we still need to use Monte Carlo methods.

\section{RESULTS}

The results reported here are for the absorption behavior of $2 \mathrm{DEG}$ at the $\mathrm{AlGaN} / \mathrm{GaN}$ interface. In an experimental measurement it is important to

$$
\begin{aligned}
& \Delta E=\frac{2 i E_{0} F_{\mathrm{ac}} q \tau_{E_{0}}\left(F_{\mathrm{dc}} q \tau_{m_{0}}+m^{*} v_{0}\left(1+i \tau_{m_{0}} \omega\right)\right) \cos \theta}{m^{*}\left(-\left(1+i \tau_{m_{0}} \omega\right)\left(-3 b k_{\mathrm{B}} T-2 E_{0}\left(1-b+i \tau_{E_{0}} \omega\right)\right)+2 a F_{\mathrm{dc}} q v_{0} \tau_{E_{0}} \cos \theta\right)} \\
& \Delta v=\frac{F_{\mathrm{ac}} q\left(\tau_{m_{0}}\left(3 b k_{\mathrm{B}} T+2 E_{0}\left(1-b+i \tau_{E_{0}} \omega\right)\right)-2 a m^{*} v_{0}^{2} \tau_{E_{0}} \cos \theta\right)}{m^{*}\left(-\left(1+i \tau_{m_{0}} \omega\right)\left(-3 b k_{\mathrm{B}} T-2 E_{0}\left(1-b+i \tau_{E_{0}} \omega\right)\right)+2 a F_{\mathrm{dc}} q v_{0} \tau_{E_{0}} \cos \theta\right)},
\end{aligned}
$$


measure only the absorption of 2DEG at AlGaN/ $\mathrm{GaN}$ interface. Figure 1 shows a schematic of an experimental setup to measure the absorption loss. As suggested in Fig. 1, to measure the total absorption of $2 \mathrm{DEG}$, we need to measure the absorption loss before and after we grow the AlGaN layer. After excluding the effects from the buffer region, it should be possible to obtain information on the free carrier absorption at the $\mathrm{AlGaN} / \mathrm{GaN}$ interface. However, the separation of the substrate might be a subtle issue for quantitative comparison with experimental results. Therefore, if we can make a nearly insulated $\mathrm{GaN}$ buffer region, where the free carrier absorption is weak in the buffer layer, we may not need to remove the substrate.

Figure 2 shows the velocity versus electric field $(v-E)$ curves of $\mathrm{AlGaN} / \mathrm{GaN}$ HFETs and AlGaAs/ GaAs HFETs calculated by the Monte Carlo method with a dc bias. From Fig. 2, we see that the $v-E$ curve of nitride HFETs has many different regions compared to other III-V materials such as GaAs. Due to the large LO polar optical phonon energy $(\sim 90 \mathrm{meV})$, there is a hump in the region $\mathrm{A}$ and $\mathrm{B}$, as shown in Fig. 2a. This is due to the sudden increase in the polar optical phonon emission scattering rate when the average electron energy exceeds the LO phonon energy under a dc bias. This effect is not seen in GaAs HFETs, as shown in Fig. 2b, since the LO phonon energy of GaAs is smaller $(\sim 34 \mathrm{meV})$ and the polar optical phonon emission scattering rate is smaller. Therefore, the $v-E$ curve of GaAs is more linear before it reaches the peak velocity. This difference causes variations in the carrier absorption behavior between the $\mathrm{GaN}$ and GaAs structures as we will see later.

Figure 3 shows the absorption loss in the 2DEG at the $\mathrm{AlGaN} / \mathrm{GaN}$ interface obtained from the EMC simulation. A 2DEG carrier density of $1 \times$ $10^{13} \mathrm{~cm}^{-2}$ is used in calculating the absorption loss. We show the results for five different $F_{\text {ac }}$ values as

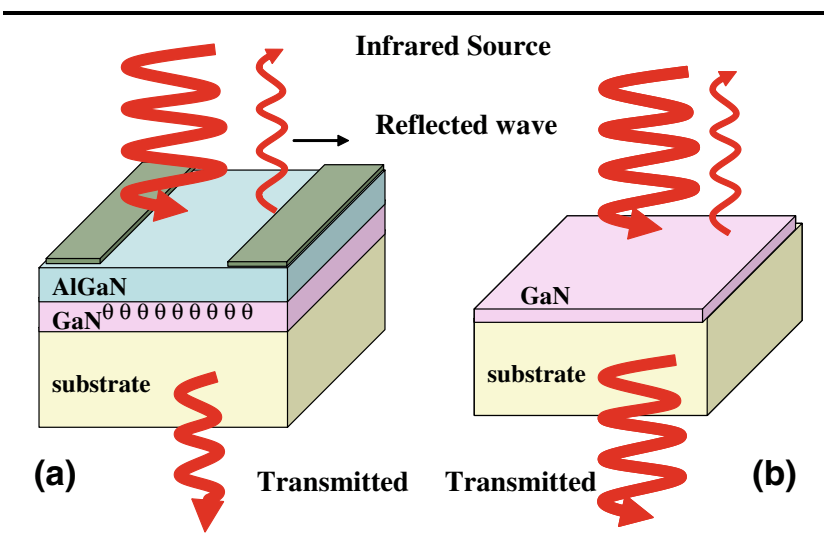

Fig. 1. A schematic of a suggested experiment setup for measuring the absorption loss of 2DEG at AIGaN/GaN interface. (a) We measure the transmission and reflection intensity $T$ and $R$, respectively, and (b) we can etch down to the active channel layer and measure the reflection and transmission intensities again to calibrate the effects of substrate and buffer region. absorption loss versus frequency. As can be seen from Eqs. 7 and 8, the absorption is strongly dependent on the mobility, which is strongly related to the strength of the electric field, as shown in Fig. 2. For larger $F_{\text {ac }}$, the carriers have a lower mobility so that the absorption losses are small at low frequencies and strong at high frequencies. On the other hand, at smaller $F_{\text {ac }}$ values, the absorption loss is strong at low frequencies and weak at high frequencies. We used Eq. 6 to fit the absorption spectrum of the AlGaN/GaN HFETs obtained from the EMC simulations. The values of the $\tau_{m}, \mu$, and $m^{*}$ parameters obtained from the fit are listed in Table I, which also lists the $\mu_{\mathrm{dc}}$ values obtained from dc Monte Carlo simulation. As shown in Table I, the fitted momentum relaxation time $\tau_{m}$ value is large for small $F_{\mathrm{ac}}$ and become much smaller at larger $F_{\text {ac }}$ values. If we obtain the effective mass $m_{\mathrm{e}}^{*}$ from the fitting, we find that the fitted effective masses increase as the ac field amplitude increases from $500 \mathrm{~V} / \mathrm{cm}$ to $50 \mathrm{kV} / \mathrm{cm}$. This can be explained by the nonparabolic band effect due to which the effective mass is larger at higher carrier
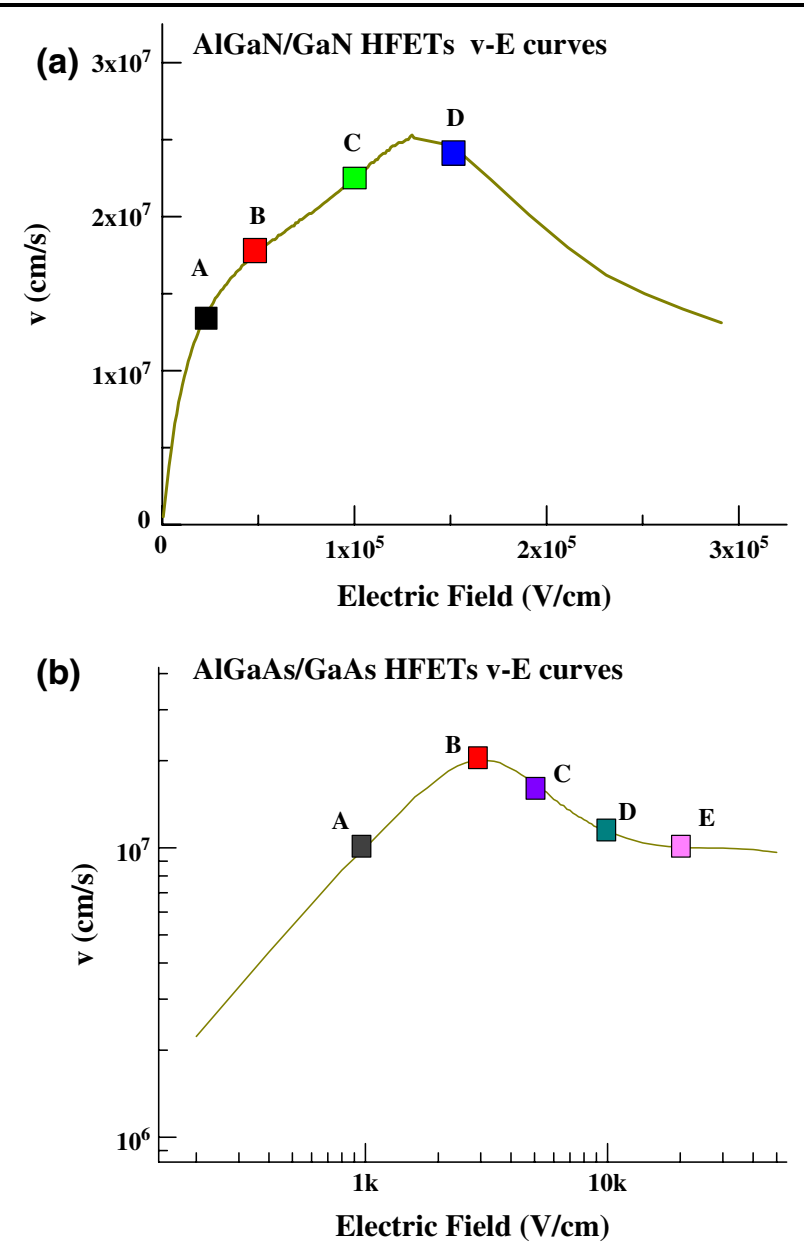

Fig. 2. The velocity field characteristics of (a) AIGaN/GaN HFETs and (b) AIGaAs/GaAs HFETs. The points A, B, C, D, and E are referred to in the text. 


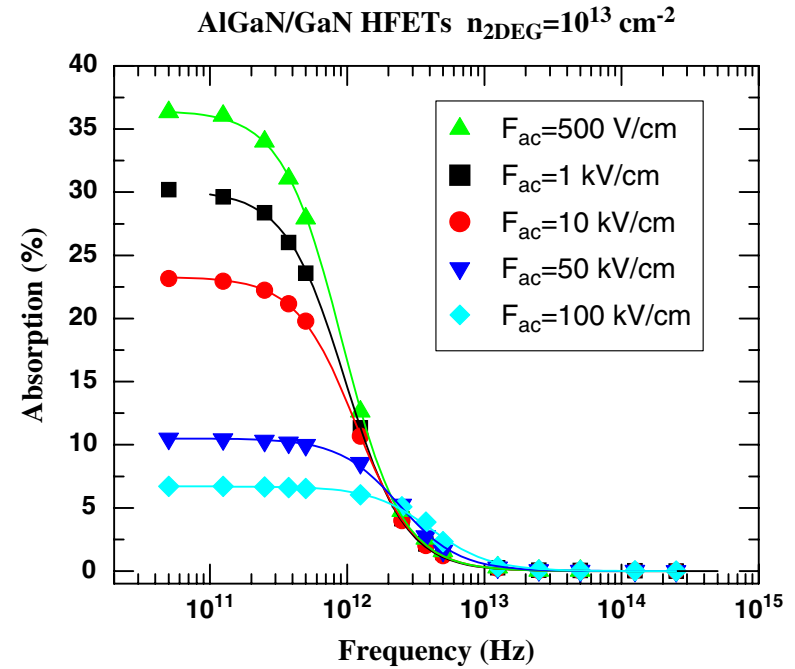

Fig. 3. The absorption loss versus infrared radiation frequency for various radiation strengths at the AIGaN/GaN interface. The sheet charge density is $1 \times 10^{13} \mathrm{~cm}^{-2}$.

energy. However, when $F_{\text {ac }}$ reaches $100 \mathrm{kV} / \mathrm{cm}$, the fitted effective mass become smaller. This is because the electron energy is high enough that intervalley scatters between the $\Gamma$ valley and the L-M valley starts and the simple Drude-Zener theory is not accurate. The $\mu_{\mathrm{dc}}$ value is the reference mobility when the dc bias is the same as the root-meansquare value of $F_{\mathrm{ac}}$. As shown in Table I, the dc and ac mobilities are quite close and therefore we can obtain a reasonable value of the dc mobility from the absorption data using the Drude model discussed.

As mentioned earlier, it is important to compare the absorption loss of AlGaN/GaN HFETs with AlGaAs/GaAs HFETs since the carrier dynamics in GaAs are quite different from those in GaN. Figure 4 shows the absorption loss of $\mathrm{AlGaAs} / \mathrm{GaAs}$ HFETs calculated by the EMC model. The AlGaAs/ GaAs HFET is modulation-doped with a sheet charge density of $1 \times 10^{12} \mathrm{~cm}^{-2}$. The parameters used for the EMC calculation can be found in Ref. 11. In the low-field region, unlike in $\mathrm{GaN}$ HFETs, we find that the absorption does not change too much before the start of intervalley scattering. The velocity peak for GaAs appears near $3 \mathrm{kV} / \mathrm{cm}$, and we find that the absorption loss changes

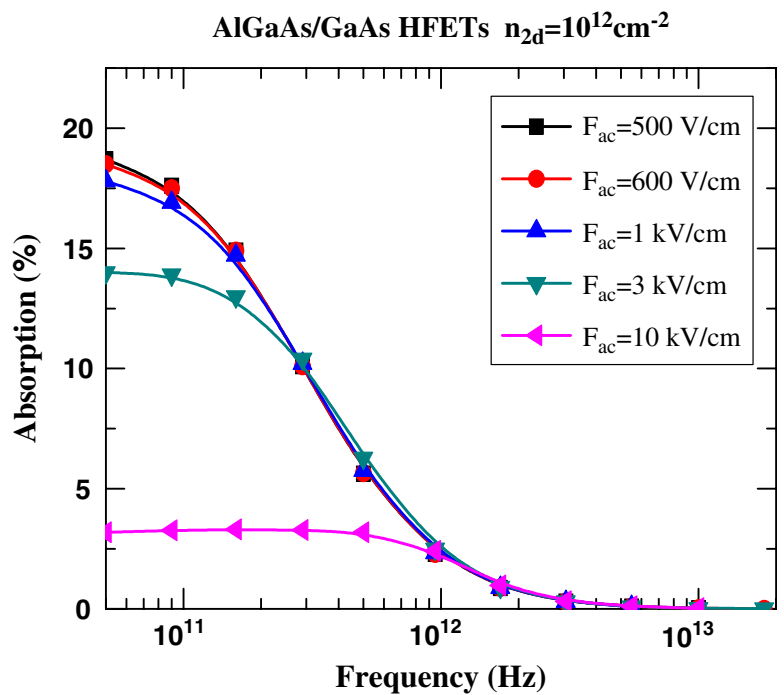

Fig. 4. The absorption loss versus infrared radiation frequency for different radiation strengths at the AIGaAs/GaAs interface. The sheet charge density is $1 \times 10^{12} \mathrm{~cm}^{-2}$.

significantly when the ac amplitude is larger than $3 \mathrm{kV} / \mathrm{cm}$. Table II shows the fitted $\tau_{m}, \mu_{\text {ac }}$, and $m_{\mathrm{e}}^{*}$ values for the AlGaAs/GaAs HFETs. As mentioned earlier, the electron mobility, $\tau_{\mathrm{m}}$, and $m_{\mathrm{e}}^{*}$ do not change too much for $E<3 \mathrm{kV} / \mathrm{cm}$. When electron intervalley scattering starts, the fitted electron effective mass increases due to the higher effective mass in the second valley. The electron relaxation time also decreases significantly and the velocity of electron goes to saturation.

As suggested in Ref. 5, the free carrier absorption spectrum under a dc bias can yield additional information on carrier dynamics. Figure 5 shows the simulated absorption loss for a low-intensity radiation source versus the frequency under different dc bias values. In Fig. 5a, the polarization of the radiation source is perpendicular to the dc bias and in Fig. $5 \mathrm{~b}$ the polarization is parallel to the dc bias. The simulations show that, when the device is under a dc bias, by measuring the absorption spectra, it is possible to extract characteristics of the carrier dynamics such as $\tau_{\mathrm{m}}$ and $\tau_{\mathrm{E}}$ without the need for strong radiation sources. The absorption spectra also show significantly different behaviors for the radiation sources parallel and perpendicular to the

Table I. The Fitted Momentum Relaxation Time and Effective Mass for AlGaN/GaN HFETs with Different Radiation Strengths

\begin{tabular}{|c|c|c|c|c|}
\hline$F_{\text {ac }}(\mathbf{k V} / \mathbf{c m})$ & $\left\langle\tau_{m}\right\rangle(\mathbf{f s})$ & $\mu\left(\mathrm{cm}^{2} / \mathrm{V} \mathrm{s}\right)$ & $m^{*}\left(m_{0}\right)$ & $\mu_{\mathrm{dc}}$ at $F_{\mathrm{ac}} / \sqrt{2}\left(\mathrm{~cm}^{2} / \mathrm{V} \mathrm{s}\right)$ \\
\hline 0.5 & 173 & 1400 & 0.217 & 1192 \\
\hline 1 & 163 & 1149 & 0.249 & 1106 \\
\hline 10 & 137 & 888 & 0.271 & 982 \\
\hline 50 & 66.5 & 396 & 0.295 & 450 \\
\hline 100 & 39.2 & 257 & 0.268 & 277 \\
\hline
\end{tabular}


Table II. The Fitted Momentum Relaxation Time and Effective Mass for AlGaAs/GaAs HFETs with Different Radiation Strengths

\begin{tabular}{|c|c|c|c|c|}
\hline$F_{\mathbf{a c}}(\mathbf{k V} / \mathbf{c m})$ & $\left\langle\tau_{\boldsymbol{m}}\right\rangle(\mathbf{f s})$ & $\mu\left(\mathrm{cm}^{2} / \mathrm{V} \mathrm{s}\right)$ & $m^{*}\left(m_{0}\right)$ & $\mu_{\mathrm{dc}}$ at $F_{\mathrm{ac}} / \sqrt{2}\left(\mathrm{~cm}^{2} / \mathrm{V} \mathrm{s}\right)$ \\
\hline 0.5 & 503 & 9775 & 0.0825 & 10,981 \\
\hline 0.6 & 497 & 9627 & 0.0826 & 10,900 \\
\hline 1 & 472 & 9300 & 0.0812 & 10,554 \\
\hline 3 & 354 & 7357 & 0.0768 & 8477 \\
\hline 10 & 124 & 1728 & 0.1148 & 1900 \\
\hline
\end{tabular}

(a) AlGaN/GaN HFET with ac field normal to de bias

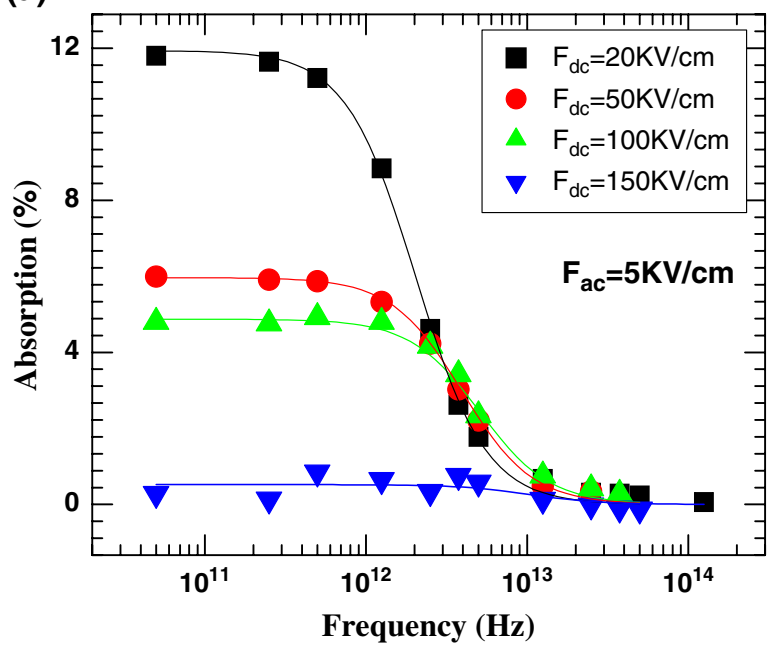

(b) AlGaN/GaN HFET with ac field parallel to dc bias

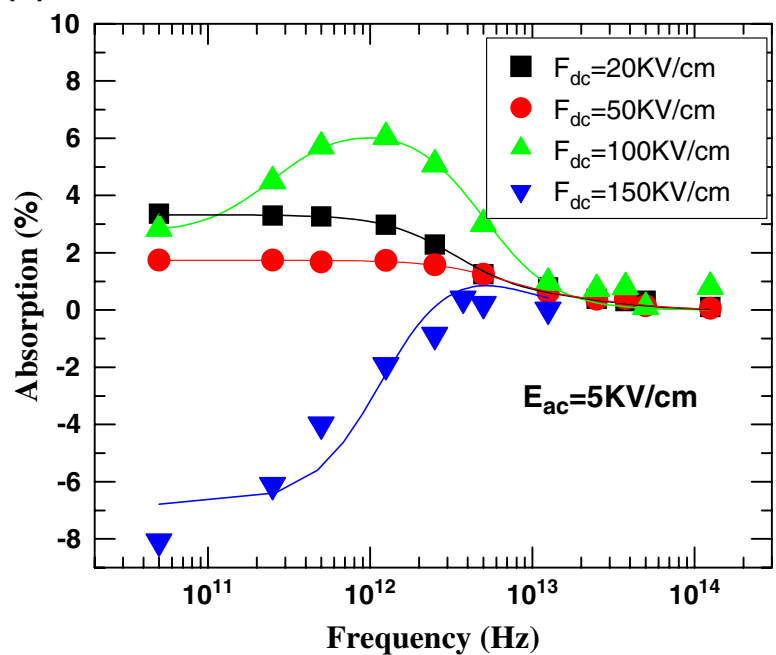

Fig. 5. The absorption loss versus infrared radiation frequency at the AIGaN/GaN interface with different values of dc bias. The infrared radiation field amplitude is $5 \mathrm{kV} / \mathrm{cm}$. The sheet charge density is $1 \times 10^{13} \mathrm{~cm}^{-2}$. (a) The polarization of the infrared radiation source is perpendicular to the dc bias, and (b) The polarization of the infrared radiation source is parallel to the dc bias.

dc bias. As shown in Fig. 5a, when the polarization is perpendicular to the dc bias, the absorption loss is given by Eq. 16, which is the same as Drude-Zener theory but with $\mu$ replaced by $\mu\left(F_{\mathrm{dc}}\right)$. When the polarization is parallel to the dc bias, there is a significant difference from the perpendicular case. The absorption loss becomes smaller than in the perpendicular case since electron transport under a dc bias is directly perturbed by the external radiation field. Table III shows the fitting values of Fig. 5. We find that $a$ and $b$ are not in a reasonable range for dc bias values smaller than $100 \mathrm{kV} / \mathrm{cm}$. This may be due to complicated phonon scattering processes. As we know, the polar optical phonon energy of $\mathrm{GaN}$ is around $91 \mathrm{meV}$, which is much larger than $3 / 2 k_{\mathrm{B}} T$ at room temperature. Therefore, there is a highly nonlinear scattering behavior since the optical phonon emission will only occur once electrons have enough energy to emit optical phonons. ${ }^{12}$ Also inter-subband scattering and the nonequivalent intervalley scattering between $\Gamma$ and L-M scattering make it even more complicated. Therefore, the simple analytical models presented in Eqs. 11 to 17 may not be able to handle these behaviors well. Only when electrons reach the saturation velocity region can this model be used. This also suggests that one needs to be careful in using the hydrodynamical model since $\tau_{\mathrm{E}}, a$, and $b$ may not be consistent for different biases.

We find that, when the dc bias is in the negative differential mobility region $\left(F_{\mathrm{dc}}=150 \mathrm{kV} / \mathrm{cm}\right)$, as shown in Fig. 2a as points $\mathrm{D}$, the absorption loss obtained from the EMC calculation is negative in the low-frequency region. This means that, instead of absorbing the energy from the radiation source, the electron emits more phonons than it absorbs from the infrared source. Of course, energy conservation holds here but the extra energy is provided by the dc bias. One possible reason is that, in the negative differential mobility region, the electron is transferring between the first $(\Gamma)$ and the second valley $(\mathrm{L}-\mathrm{M})$. The perturbation of the small parallel electric field will assist the electron to release the energy and jump back to the lower valley. This behavior is similar to that seen in an inter-subband laser, where there is carrier inversion and the incident light stimulates electrons to emit light and jump to a lower subband. These effects are not seen in $\mathrm{Si}$ since there is no negative differential mobility region.

Figure 6 shows the simulated absorption loss of GaAs HFETs; it is important to compare this with GaN HFETs. In Fig. $6 a$ and b, the polarization of radiation source is perpendicular and parallel to the 
Table III. The Fitted Momentum and Energy Relaxation Time and Effective Mass for AlGaN/GaN HFETs Under Various de Biases

\begin{tabular}{|c|c|c|c|c|c|c|}
\hline$\overline{F_{\mathrm{dc}}(\mathrm{kV} / \mathrm{cm})}$ & $\left\langle\tau_{\mathbf{m}}\right\rangle(\mathbf{f s})$ & $\mu\left(\mathrm{cm}^{2} / \mathrm{Vs}\right)$ & $m^{*}\left(m_{0}\right)$ & $\left\langle\tau_{E}\right\rangle(\mathbf{f s})$ & $a$ & $\bar{b}$ \\
\hline 20 & 78.03 & 458 & 0.299 & 31.48 & 27.14 & -40.30 \\
\hline 50 & 40.48 & 229 & 0.311 & 25.18 & 12.66 & -18.37 \\
\hline 100 & 31.44 & 187 & 0.232 & 46.36 & 0.02789 & 1.283 \\
\hline 150 & 26.8 & 156 & 0.300 & 272.7 & -2.998 & 0.30 \\
\hline
\end{tabular}

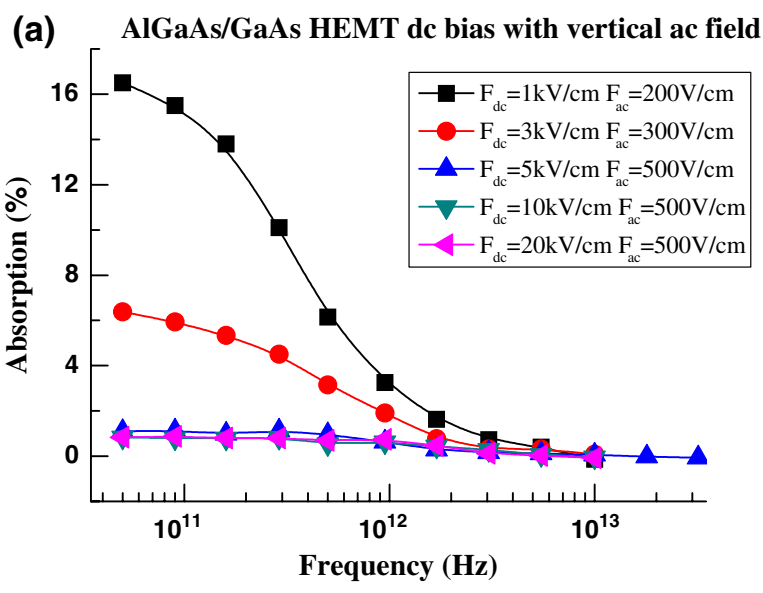

(b) AlGaAs/GaAs HFET de bias with parallel ac field

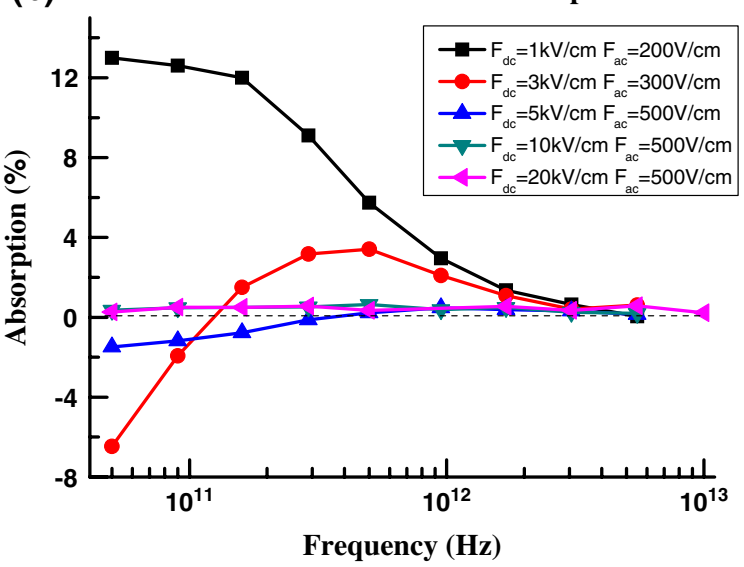

Fig. 6. Absorption loss versus infrared radiation frequency at the AlGaAs/GaAs interface with different dc biases. The sheet charge density is $1 \times 10^{12} \mathrm{~cm}^{-2}$ : (a) with the polarization of the infrared radiation source perpendicular to the dc bias, and (b) with the polarization of the infrared radiation source parallel to the dc bias.

dc bias, respectively. As shown in Fig. 6a, when the polarization of the infrared source is perpendicular to the dc bias, the behavior is very similar to that seen in GaN HFETs and we can extract $\tau_{\mathrm{m}}$ and $\mu\left(F_{\mathrm{dc}}\right)$ directly from the absorption spectrum. In Fig. $6 \mathrm{~b}$, we see that the absorption behavior is quite different from the perpendicular case. We again observe the negative absorption loss when the electric field is $3 \mathrm{kV} / \mathrm{cm}$ and $10 \mathrm{kV} / \mathrm{cm}$. As shown in Fig. $2 b$, the differential mobility is negative at these field values. The absorption becomes positive again when the velocity goes to saturation. The simulation shows that it is important to examine these effects experimentally since it might be a good high-frequency radiation source.

\section{CONCLUSION}

In this paper we have carried out a generalization of the Drude free carrier absorption model. We find through Monte Carlo simulation that free carrier absorption under conditions of dc bias and with ac polarization parallel and normal to the dc field can provide very detailed carrier dynamic information. In GaN HFETs, the $v-E$ curve has several regions: linear, shoulder region, peak velocity, and negative differential resistance, all of which are reflected in the free carrier absorption.

\section{ACKNOWLEDGEMENTS}

This work has been supported by a DARPA MURI program.

\section{REFERENCES}

1. M. Higashiwaki, T. Matsui, and T. Mimura, in Device Research Conference (State College, PA, USA, 2006), pp. 149-150.

2. T. Palacios, A. Chakraborty, S. Heikman, S. Keller, S.P. Denbaars, and U.K. Mishra, IEEE Electron. Dev. Lett. 27, 13 (2006).

3. Y.-R. Wu and J. Singh, J. Appl. Phys. 101, 113712 (2007).

4. A. Matulionis, J. Liberis, L. Ardaravicius, L.F. Eastman, J.R. Shealy, and A. Vertiatchikh, Semicond. Sci. Technol. 19, S421 (2004).

5. H. Jiang, J.M. Hinckley, and J. Singh, IEEE J. Quantum Electron. 33, 1779 (1997).

6. T.-R. Tsai, S.-J. Chen, C.-F. Chang, S.-H. Hsu, T.-Y. Lin, and C.-C. Chi, Opt. Exp. 14, 4898 (2006).

7. H. Tiedje, H. Haugen, and J. Preston, Opt. Commun. 274 , 287 (2007).

8. F.K. Reinhart, J. Appl. Phys. 97, 123536 (2005).

9. Y.R. Wu, M. Singh, and J. Singh, IEEE Trans. Electron. Dev. 53, 588 (2006).

10. I. Vurgaftman, J.R. Meyer, and R.L.R. Mohan, J. Appl. Phys. 89, 5815 (2001).

11. J. Singh, Physics of Semiconductors and their Heterostructures (McGraw-Hill, Inc., 1993).

12. Y.R. Wu, M. Singh, and J. Singh, IEEE Trans. Electron. Dev. 52, 1048 (2005). 